\title{
Finite Element Modeling of the behavior of a hollow cylinder in a hydrogen-containing environment
}

\author{
A. A. Lakhdari \\ Université des Sciences et de la Technologie d'Oran Mohamed Boudiaf, Algeria \\ amina.lakbdari@univ-usto.d₹, bttps:/ / orcid.org/0000-0003-0627-0194
}

\author{
S.A. Bubnov \\ Ryazan State Radio Engineering University, Russia \\ serbubnov@inbox.ru, bttps://orcid.org/0000-0002-0274-8341
}

\author{
A. Seddak \\ Université des Sciences et de la Technologie d'Oran Mohamed Boudiaf, Algeria \\ sed_dz@yahoo.fr, bttps://orcid.org/0000-0003-4539-8350
}

\author{
I. I. Ovchinnikov, I. G. Ovchinnikov \\ Saratov State Technical University Gagarin Y.A., Russia \\ bridgear@@mail.ru, bttp:// orcid.org/0000-0001-8370-297X \\ bridgesan@mail.ru, bttps://ordic.org/0000-0003-0617-3132
}

\begin{abstract}
The two main research orientations on the problem of hydrogen embrittlement are examined: the study of fundamental principles and the disclosure of micromechanisms and the relation between hydrogen embrittlement and metal aging; the development of models and methods for predicting the kinetics of change in stress-strain state and for evaluating the longevity of structures subjected to hydrogen embrittlement. The state of the problem of hydrogen embrittlement of metals in the first direction is briefly analyzed. More attention is paid to the importance of predicting the behavior of charged metal structures under the influence of hydrogen embrittlement. We then examine the use of finite element modeling using the ANSYS software to compute the calculation analysis of a hollow cylinder subjected to internal and external pressures and hydrogen embrittlement. The cylinder material is nonlinear elastic and its properties depend on the hydrogen concentration at each point of the cylinder. Consideration is given to the influence of the rigidity of the stress state and the hydrogen concentration on the diffusion kinetics of hydrogen in the cylinder body. The problem is solved in time steps. The distributions of the hydrogen concentration and the stresses for a quarter of the volume of the cylinder are given, as well as the
\end{abstract}

\section{OPEN ACCESS}

Citation: A. A. Lakhdari, S.A. Bubnov, A. Seddak, I. I. Ovchinnikov, I. G. Ovchinnikov, Finite Element Modeling of the behavior of a hollow cylinder in a hydrogen-containing environment, Frattura ed Integrità Strutturale, 51 (2020) 236-253.

Received: 06.10 .2019

Accepted: 26.11 .2019

Published: 01.01.2020

Copyright: (C) 2020 This is an open access article under the terms of the CC-BY 4.0, which permits unrestricted use, distribution, and reproduction in any medium, provided the original author and source are credited. 
graphs of these values according to the thickness of the wall of the cylinder at different times.

It is shown that the ANSYS software package adapted to the resolution of such problems can model the behavior of different structures in a hydrogencontaining environment, taking into account the effects caused by both the influence of hydrogen on mechanical properties of the material and by the stress state of the structures, as well as by the influence of the stress state on the interaction kinetics of hydrogen with the structures.

KEYwORDS. Hydrogen embrittlement; Hollow cylinder; Physical nonlinearity; Hydrogen effect; Finite element modeling.

\section{INTRODUCTION}

he influence of hydrogen on the mechanical properties of structural material is a topical problem, because in recent times hydrogen is widely used in various industrial fields. Hydrogen has a destructive effect on materials and structures at elevated temperatures and pressures, as well as at normal temperatures known as conventionally low temperatures [1-6].

At the present time, the term "embrittlement by hydrogen" is understood to include all the detrimental influence of hydrogen on the properties of materials.

Hydrogen embrittlement (HE) is a direct consequence of a critical local hydrogen concentration in a metallic material. There are two main mechanisms of hydrogen-assisted rupture [7-9]:

- HEDE (Hydrogen-Enhanced DEcohesion);

- HELP (Hydrogen-Enhanced Localised Plasticity).

The hypothesis of the HEDE mechanism is based on the favoring of the formation of microcracks following the reduction of the cohesion of the metal network, that is to say on the weakening of the inter-atomic bonds, following a high concentration of hydrogen in crack tip. In contrast, the HELP mechanism is based on the ductile nature of hydrogen assisted rupture. The HELP mechanism involves hydrogen-plasticity interactions of an elastic nature. The HELP model represents variations in crack tip plasticity and the HEDE model explains the speed of propagation of microcracks.

There is also another Adsorption Induced Dislocation Emission (AIDE) model, which suggests, as with the HEDE mechanism, that hydrogen embrittlement is due to the weakening of inter-atomic bonds.

As is known, hydrogen interacts differently with metals, depending on the temperature and pressure exerted on the structure.

The introduction of hydrogen into metals and alloys can take place by one of two qualitatively different mechanisms [10]:

1.As a result of low temperature electrochemical processes with the participation of hydrogen ions, which are reduced and absorbed by steel. At low temperatures (i.e. ordinary temperatures), hydrogenation of the metal occurs, resulting in embrittlement by hydrogen and modification of the mechanical properties of the metal. This process is often called low temperature hydrogen embrittlement.

2. From a hydrogenated gaseous medium, at temperatures (above $200^{\circ} \mathrm{C}$ ) and high pressures, as a result of thermal dissociation of hydrogen molecules, forming atomic hydrogen absorbed by steel, which interacts with carbides. High temperature hydrogen corrosion occurs which destroys the material of the structural elements. Many studies have been devoted to the problem of high temperature hydrogen corrosion of metal structures [7-10]. This process is called high temperature hydrogen corrosion.

According to the work [11], there are two main lines of research on low-temperature hydrogen embrittlement:

a) study of the fundamentals of hydrogen embrittlement processes ;

b) development of deformation models and estimation of the longevity of structural elements interacting with hydrogen.

The first axis has been studied and analyzed in sufficient detail in many studies and it has been found that the processes of hydrogen embrittlement and degradation of the mechanical properties of pipeline materials were not sufficiently studied [12-16]. 
The development of deformation models and the evaluation of the longevity of structural elements subjected to hydrogen embrittlement have been the subject of several studies [11], and in this work the finite difference method was generally used to calculate the structures.

In the work [17], the process of hydrogenation and deformation of a hollow cylinder was studied, taking into account the effect of the stress state on the hydrogen saturation kinetics of the structure.

In this work, we examine finite element modeling of the behavior of a long hollow cylinder under hydrogenation conditions, taking into account the effect and concentration of hydrogen as well as the rigidity of the flow diagram state of stress on the kinetics of penetration of hydrogen into the cylinder.

\section{LOW TEMPERATURE HYDROGEN EMBRITTLEMENT}

$\mathrm{I}$ $\mathrm{t}$ is observed at temperatures below $200^{\circ} \mathrm{C}\left(-20\right.$ to $\left.200^{\circ} \mathrm{C}\right)$, and in this case, the source of hydrogen is either hydrogen itself, or hydrogen is a by-product in some technological processes, so hydrogen enters the metal simply under pressure.

The effect of hydrogen at low temperature is distinguished by the penetration of hydrogen by diffusion in the elements of metal structures under constraints or not. In addition, the hydrogen penetrates intensively in the zones under traction, and much less in the zones under compression. Thus, it accumulates and after reaching a certain concentration, it causes a change in the mechanical properties of the material of the structure, depending on its concentration. For a low concentration of hydrogen, there is practically no change in the mechanical properties.

But by reaching a critical concentration level, the hydrogen causes an intense degradation of the properties, and for a maximum concentration (saturation limit), the change of the mechanical properties is slowed down, although the hydrogen saturation of the material continues $[17,18]$.

In the structures under stress and subjected to a hydrogenation at low temperature, there is a more intense variation of the mechanical properties of the material in the zones under traction than in the other zones under compression.

The non-uniform variation of the mechanical properties causes a redistribution of the stress field, which in turn influences the distribution of the hydrogen concentration. The redistribution of the stresses and the hydrogen field in the mass of the structural element will remain unstable as long as the state of the structure is not stabilized.

During the low temperature hydrogenation, a physico-chemical interaction of the steel with the hydrogen takes place, leading to an irreversible change of the mechanical properties. This is mainly due to the destruction of the carbide component. This physico-chemical phenomenon is called hydrogen corrosion of steel.

Hydrogen corrosion develops in carbon steels after long operation at high temperature and pressure in an environment containing hydrogen. At the base of the mechanism of hydrogen corrosion is the interaction of hydrogen with carbon with the formation of methane. This reaction begins with the decarburization of the surface and the formation of microcracks, which progressively propagate in the metal, reducing its strength and plasticity.

Hydrogen embrittlement of metal structures is closely related to their microstructure, and in particular to the processes of segregation and diffusion occurring at interfaces and defects.

\section{FORMULATION OF THE PROBLEM}

he behavioral model of a long hollow cylinder under low temperature hydrogen embrittlement conditions is examined, in which hydrogen, without chemical interaction with the metal, penetrates into the volume of the structural element and accumulates there.

It is accepted that the penetration of hydrogen takes place by the diffusion mechanism. In this case, as the experimental data show, the hydrogen does not penetrate equally in the different zones of the structural element in the zones where the compressive stresses predominate, the hydrogen penetrates more slowly, in the zones where tensile stresses are predominant, hydrogen penetrates faster. Thus, we have a medium whose diffusion characteristics depend on the state of stress $[17,18]$.

Also according to experimental data, the diffusion characteristics of the cylinder material depend on the hydrogen concentration.

Therefore, it is further assumed that the diffusion coefficient of hydrogen in structure $D(C, S)$ depends on the rigidity of the state of stress $S$ and the hydrogen concentration $C$ :

$$
D(C, S)=D_{0} \cdot(1+\alpha C+\beta S)
$$


where:

$D$ - diffusion coefficient, without taking into account the effect of hydrogen in not charged structure;

$\alpha$ and $\beta$-coefficients to be determined based on experimental data;

$C$ - hydrogen concentration;

$S$ - parameter, characterizing the rigidity of the state of stress schema, with:

$$
\mathrm{S}=\sigma_{\mathrm{o}} / \sigma_{\mathrm{i}}
$$

where:

$\sigma_{o}$ - mean stress;

$\sigma_{i}$ - intensity of the constraints.

According to formula (1), with increasing hydrogen concentration, the diffusion coefficient also increases. The $S$ parameter takes negative values, if the compression components predominate in the constraint state schema. In this case, the diffusion coefficient decreases. On the other hand, with a predominance of traction components, the parameter $S$ takes positive values, which leads to an increase in the diffusion coefficient.

We consider that the structural element consists of a nonlinear elastic material whose deformation diagram is approximated by the function:

$$
\sigma_{\mathrm{i}}=A \cdot \varepsilon_{\mathrm{i}}-B \cdot \varepsilon_{\mathrm{i}}^{\mathrm{m}}
$$

where:

$A, B$ and $m$ - known coefficients;

$\varepsilon_{i}$ - intensity of deformations.

To take into account the influence of hydrogen on the deformation diagram, by analogy with [16, 17], we use an influence function $\theta(C, S)$ :

$$
\sigma_{\mathrm{i}}=A \cdot \varepsilon_{\mathrm{i}}-B \cdot \varepsilon_{\mathrm{i}}^{\mathrm{m}} \cdot \theta(C, S)^{\mathrm{m}-1}
$$

where:

$$
\begin{gathered}
1-\text { then } S<S_{0} \\
\theta(C, S)=\exp \left(-k \cdot C^{a} \cdot\left(S-S_{0}\right)^{b}\right)-\text { then } S \geqslant S_{0}
\end{gathered}
$$

In these formulas:

$S_{0}$ - a threshold value of parameter $S$, to which hydrogen does not affect the material deformation diagram;

$\mathrm{k}, \mathrm{a}, \mathrm{b}$ - known coefficients.

The modeling work involves analyzing the redistribution of stresses in the volume of a structural element as a result of low-temperature hydrogen corrosion, as well as identifying the most dangerous modes of operation.

\section{IMPLEMENTATION OF THE MODEL IN THE ANSYS FINITE ELEMENT SOFTWARE PACKAGE.}

o solve this problem, we chose the ANSYS software implementing the finite element method (FEM) [19-22 ].

The idea of the approach is that in ANSYS, each finite element (EF) can define its own properties. For example, when solving a structural problem, each finite element can be assigned an individual elasticity modulus, a transverse strain coefficient or points of the strain diagram. With the ADPL language, built into ANSYS, we have written a number of macros, which solved this problem.

All model parameters are conventionally divided into groups:

- the geometrical parameters of the hollow cylinder (inner and outer radii, length);

- the loading parameters of the structural element (internal and external pressures, hydrogen concentrations on the internal and external surfaces, number of time steps); 
- the parameters of the initial mechanical properties of the material (modulus of elasticity, transverse deformation coefficient, diffusion coefficient, density, stress diagram, etc.);

- the parameters of the finite element model which determine the quality of the mesh;

- the massive parameters, containing the mechanical properties of the material that vary in time and space. There are also massive-parameters for storing the results of calculations. In Tab. 1, the names and dimensions of the massifs used are presented.

\begin{tabular}{|c|c|c|}
\hline $\begin{array}{l}\text { Name of the } \\
\text { massif }\end{array}$ & Content & Dimension \\
\hline psy_func & $\begin{array}{l}\text { values of the function } \Psi(\varepsilon, C, S) \text { for } \\
\text { each } F E \text { at each time step }\end{array}$ & $n \times e l \_a m o u n t$ \\
\hline nu_func & $\begin{array}{l}\text { values of the function } v\left(\varepsilon_{i}, C, S\right) \text { for } \\
\text { each } F E \text { at each time step }\end{array}$ & $n \times e l \_a m o u n t$ \\
\hline teta_func & $\begin{array}{l}\text { values of the function } \theta(C, S) \text { for } \\
\text { each FE at each time step }\end{array}$ & $n \times e l \_a m o u n t$ \\
\hline strain_stress & $\begin{array}{l}\text { Values of deformations and } \\
\text { corresponding stresses under the } \\
\text { influence of hydrogen }\end{array}$ & $n \times e l \_a m o u n t$ \\
\hline$s_{-}$factor & $\begin{array}{c}\text { Parameter values, characterizing the } \\
\text { rigidity of the constraint state } \\
\text { diagram for each EF at each time } \\
\text { step }\end{array}$ & $n \times e l \_a m o u n t$ \\
\hline d_factor & $\begin{array}{l}\text { Diffusion coefficient values for each } \\
\text { FE at each time step }\end{array}$ & $n \times$ el_amount \\
\hline strain_int & $\begin{array}{l}\text { Deformation intensity values for } \\
\text { each FE at each time step }\end{array}$ & $n \times e l \_a m o u n t$ \\
\hline stress_int & $\begin{array}{l}\text { Constraint intensity values for each } \\
\text { FE at each time step }\end{array}$ & point $\times$ el_amount $\times n$ \\
\hline stress_avg & $\begin{array}{l}\text { Values of the average stress for each } \\
\text { FE at each time step }\end{array}$ & $n \times e l \_a m o u n t$ \\
\hline concentr & $\begin{array}{l}\text { Hydrogen concentration values for } \\
\text { each FE at each time step }\end{array}$ & $n \times e l \_a m o u n t$ \\
\hline
\end{tabular}

Table 1: Names and dimensions used in mass calculations

In Tab. 1:

$n$ - number of time steps needed to solve the problem;

el_amount - total number of finite elements of the model;

point - number of points by which the deformation diagram is constructed.

The massive strain_stress is three-dimensional. It is therefore possible to save a deformation diagram for each FE at each time step.

The solution of the problem is conveniently divided into several stages:

Step 1: Construction of the geometric model of the structural element.

At this stage, the construction of a geometric model of a fragment of the long hollow cylinder.

The construction of the geometric model was carried out according to the bottom-up principle, that is to say, first, the key points are defined, and then the lines passing through these points are drawn. According to the lines, was built the axial section of the cylinder. Then, by rotating the section around the OZ axis, at a 90 degree angle, is built a volume - cylinder fragment (in order to save the memory of the computer). The section could be rotated 360 degrees, to obtain the entire cylinder. For the construction of the geometric model, the cylindrical coordinate system was chosen.

Step 2: Construction of the finite element model

As is known, the use of an ordered mesh makes it possible to obtain more precise solutions, with respect to a disordered mesh. To build an ordered mesh, you have to prepare a model. For this, each line forming part of the volume must be 
divided into a determined number of segments. For the axial, radial and angular directions, the number of segments is given as parameters $n_{-} a x i s, n_{-}$tang and $n_{-}$thickness. By modifying the values of these parameters, it is possible to easily enlarge or refine the mesh of finite elements in the whole of the structural element.

When applying the mesh of finite elements, it is important to have on several thicknesses of the wall of the cylinder (not less than 10), because as a result of the influence of the hydrogenated medium will have changes in the mechanical properties, including the thickness of the cylinder. In this case, follow the form EF. Elements that are too thin and elongated may give a greater error. In case of gross violation of the geometry of the finite element, ANSYS will issue a warning. That is why, by increasing the number of elements according to the thickness, it is desirable to increase it and according to other directions.

To solve the problem, it took three types of finite element from the ANSYS library. For the diffusion problem, was chosen element SOLID239, for the structural problem - SOLID95, and for the mesh of finite elements - MESH200. All element types have a modification of 20 nodes (hexahedra), so changing the element type to solve the corresponding problem does not add any difficulties - all the nodes of the finite element model remain in place.

The finite element SOLID95 was chosen because it supports the material, for which a deformation diagram can be given. At present, this type of element is considered obsolete, but it is suitable for the resolution of some problems. The number of points on the deformation diagram must not exceed 100.

Step 3: Load application and problem resolution

At this point, the initial conditions and limits are defined and the problem is solved.

To construct the initial deformation diagram (before the effect of hydrogen, $\theta(C, S)=1$ ), we need the values of stress strain intensities, which will calculate the stress intensities according to the equation (4). This is why, first of all, the problem of Blade is solved for a hollow cylinder in linearly elastic material for the pressures (external and internal), which we will use later in calculations under the action of hydrogen. The initial strain diagram is shown in Fig. 1.

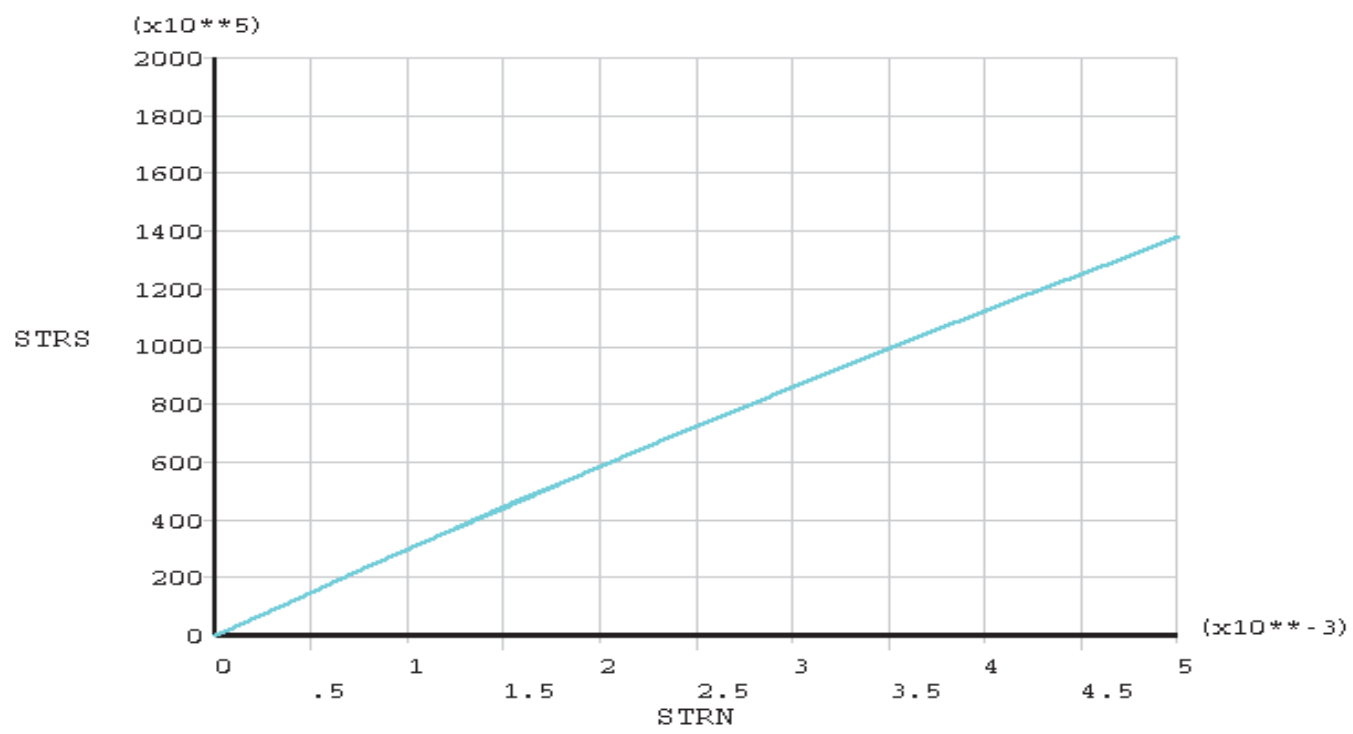

Figure 1: Initial diagram of material deformation

The solution of the problem occurs according to the following algorithm:

1. Define the boundary conditions and determine the initial stress-strain state of the structural element. Determine the value of the $S$ parameter for each finite element.

2. Define the boundary conditions for the diffusion equation and determine the initial value of the hydrogen $C$ concentration for each finite element.

3. Perform a new calculation:

- the diffusion coefficient $D$ for each finite element according to relation (1);

- points of the deformation diagram for each finite element according to relation (4). 4. Definite the boundary conditions corresponding to a given time step for the diffusion equation and its resolution with new values of the diffusion coefficient for each finite element.

5. Define the boundary conditions corresponding to a given time step and determine the new stress-strain state and the new values of the $S$ parameter for each finite element. 
6. Incremental time step.

7. Repeat steps 3-6 until the destruction of the structural element.

Fig. 2 shows the deformation diagram, constructed taking into account the effects of low temperature hydrogen.

Step 4: Results analysis.

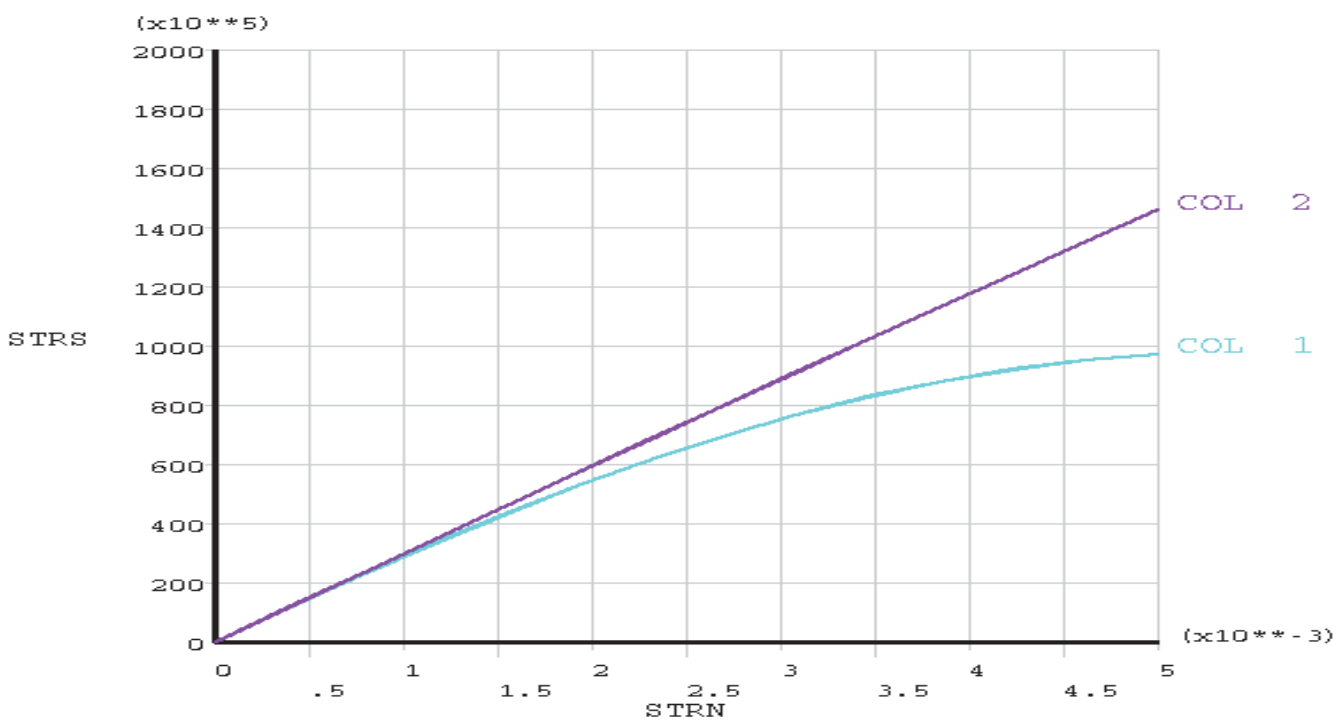

Figure 2: Initial deformation diagram (COL2) and modified by the influence of hydrogen (COL1).

\section{CALCULATION AND ANALYSIS OF THE RESULTS}

alculations were made for a hollow cylinder with the parameters: inner radius of $0.3 \mathrm{~m}$; outer radius of $0.5 \mathrm{~m}$; cylinder length of $3.5 \mathrm{~m}$. The characteristics of the material: modulus of elasticity $\mathrm{E}=3.0 \mathrm{e} 10$; transverse deformation coefficient $\mathrm{Nu}=0.2$; thermal conductivity coefficient Alpha $=1.4 \mathrm{e}-5$; density of the material Dens $=7659$; coefficients of the function of approximation of the deformation diagram: $\mathrm{A}_{0}=3.0 \mathrm{e} 10, \mathrm{~B}_{0}=2.0 \mathrm{e} 11$. The modulus of elasticity, the parameters of the strain diagram and all the constraints on the figures are measured in $\mathrm{Pa}$ (Pascal), the time is measured in hours. Tab. 2 shows the different loading cases of the hollow cylinder.

\begin{tabular}{ccccc}
\hline № Loading & $P_{\text {in }}, \mathrm{MPa}$ & $P_{\text {out }}, \mathrm{MPa}$ & $C_{\text {in }}, 1 / \mathrm{m}^{3}$ & $C_{\text {out }}, 1 / \mathrm{m}^{3}$ \\
1. & 0 & 20 & $3.2 \mathrm{E}-4$ & $3.2 \mathrm{E}-4$ \\
2. & 0 & 20 & $7.2 \mathrm{E}-4$ & $1.5 \mathrm{E}-4$ \\
3. & 0 & 30 & $7.2 \mathrm{E}-4$ & $1.5 \mathrm{E}-4$ \\
4. & 20 & 11 & $0.5 \mathrm{E}-4+0.00005 \cdot t$ & $3.2 \mathrm{E}-4$ \\
\hline
\end{tabular}

Table 2: Loading cases and effects of hydrogen

Loading №1. The hollow cylinder is under external pressure $P_{\text {out }}$, the concentration field of the hydrogen is homogeneous.

The concentration field has the form shown in Fig. 3, and it does not change over time. The intensity of the constraints is shown in Fig. 4. The radial, tangential and axial stresses at time $t=10$ are shown in Figs 5, 6, 7.

Fig. 8 shows the stress intensity curves $\mathrm{S}_{\mathrm{INT}}$, axial stresses $\mathrm{S}_{\mathrm{Z}}$, radial and tangential $\mathrm{S}_{\mathrm{X}}$ and $\mathrm{S}_{\mathrm{Y}}$ according to the thickness of the wall of the cylinder. 


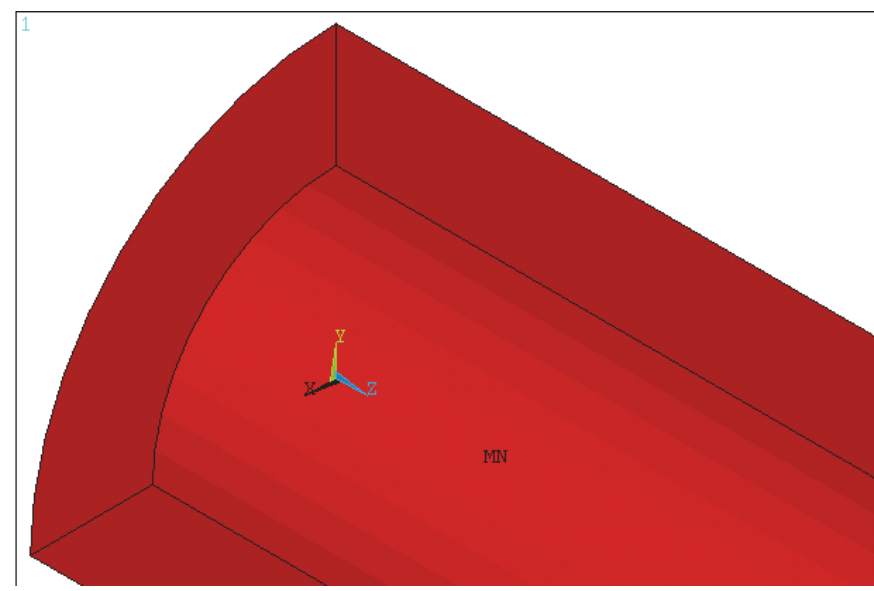

ANSYS Release 18.0

Build 18.0

$11: 35: 30$

AVG ELEMENT SOLUTION

STEP $=1$
SUB $=1$

TIME $=10$

CONCT (AVG)

$\mathrm{SMN}=.320 \mathrm{E}-03$
$\mathrm{SMX}=.320 \mathrm{E}-03$

Figure 3: Concentration field of hydrogen.

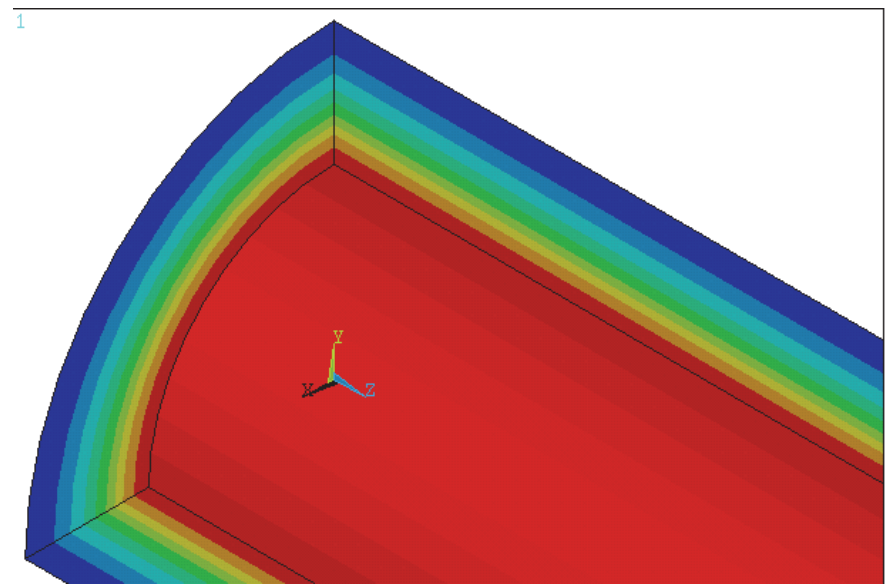

ANSYS Release 18.0

Build 18.0

20.26 .58

AVG ELEMENT SOLUTION

STEP $=1$
SUB $=1$

TIME $=10$

SIG_I (AVG)

STM $=.433 \mathrm{E}+08$

$.433 \mathrm{E}+08$
$.450 \mathrm{E}+08$

$.450 \mathrm{E}+08$
$.468 \mathrm{E}+08$

$.468 \mathrm{E}+08$
$.503 \mathrm{E}+08$

$.520 \mathrm{E}+08$
$.538 \mathrm{E}+08$

$555 \mathrm{E}+08$

$.590 \mathrm{E}+08$

Figure 4: Intensity of constraints at the moment $t=10$.

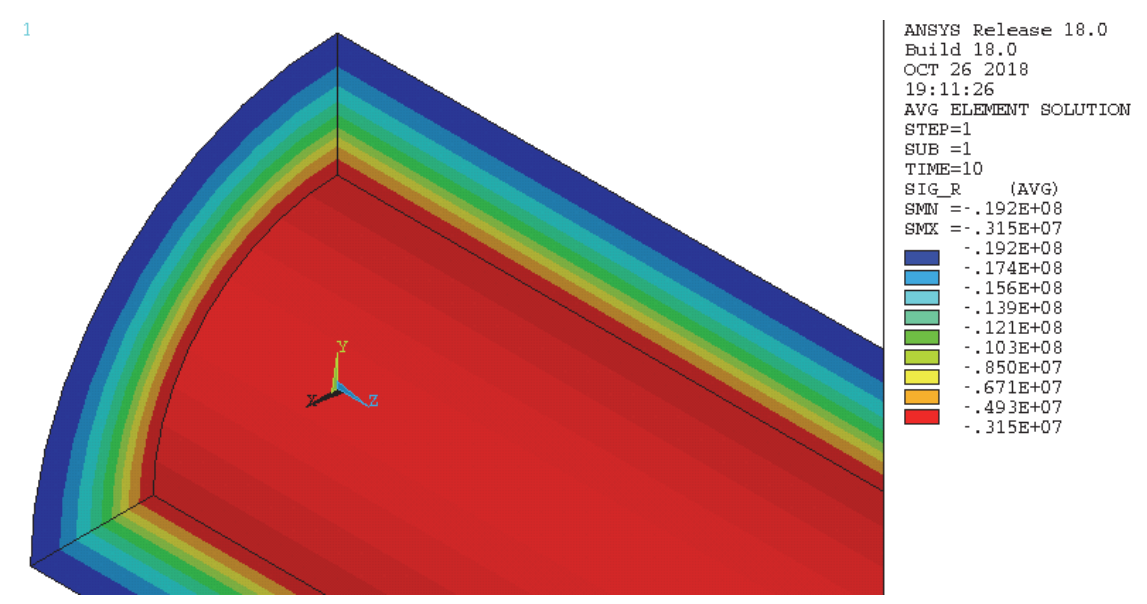

Figure 5: Radial constraints at the moment $t=10$. 


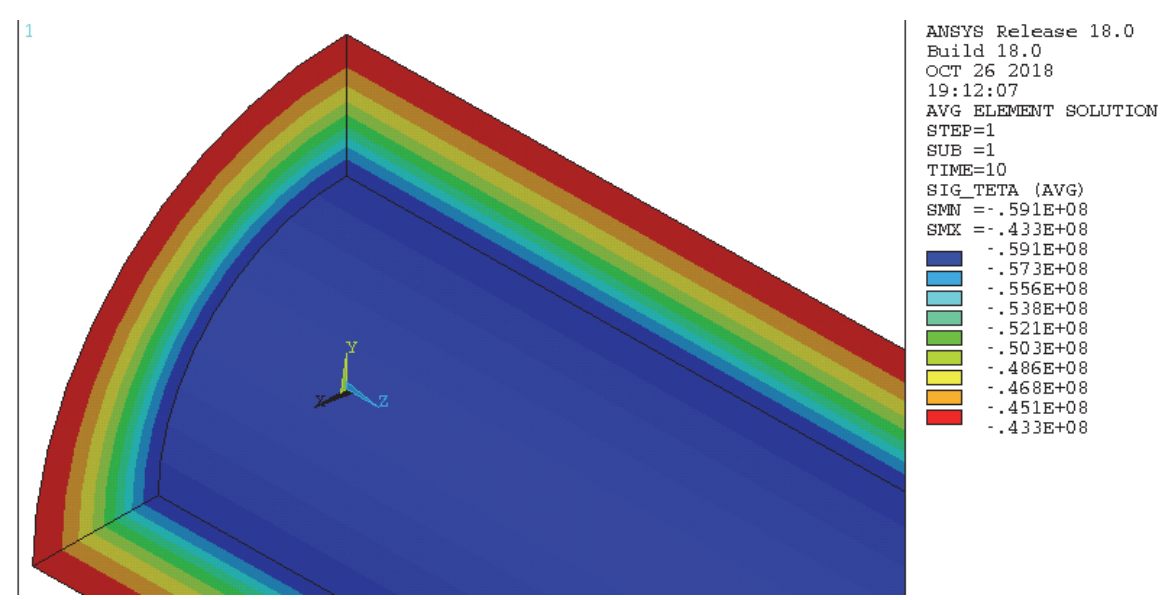

Figure 6: Tangential constraints at the moment $t=10$.

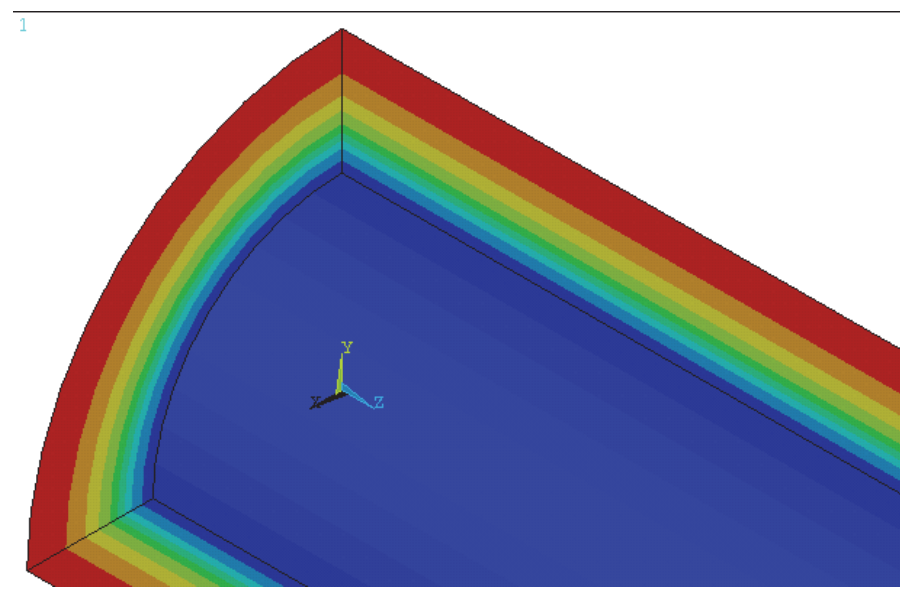

ANSYS Release 18.0

Build 18.0

OCT 262018

$19: 12: 45$
AVG ELENENT SOLUTION

$\mathrm{STEP}=1$

SUB $=1$
TINE $=10$

SIG_Z (AVG)

SINN $=-19473$.

SMX $=-3320.1$

-17678.5
-15883.7
-14088.9

\begin{tabular}{rr}
-14088.9 \\
-12294.1 \\
-20499.3 \\
\hline
\end{tabular}

$-6909.74$

-5114.96
-3320.17

Figure 7: Axial constraints at the moment $t=10$.

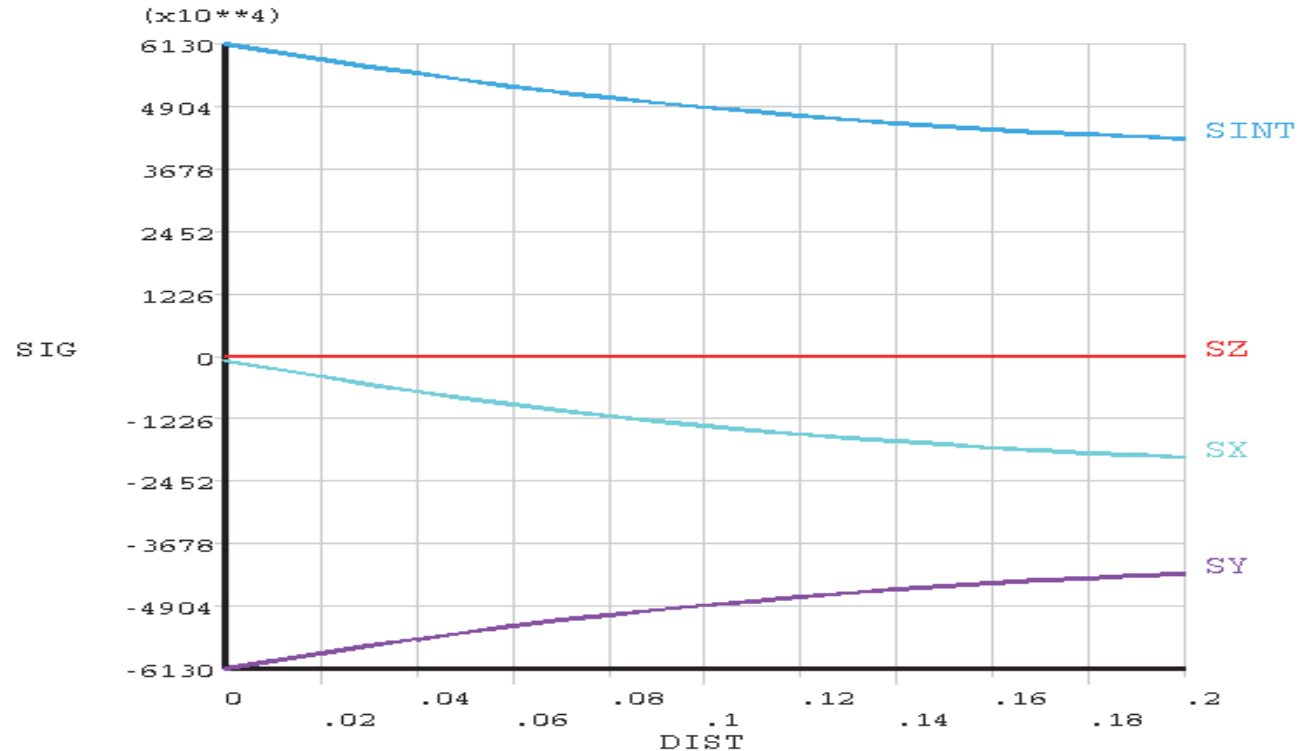

Figure 8: Stress curves according to the thickness at the moment $t=10$. 
Calculations show that there is no redistribution of stresses because the hydrogen concentration does not change in both time and volume of the structural element. As a result, hydrogen has virtually no effect on the stress state of the structural element.

Loading №2. The hollow cylinder is under external pressure $\mathrm{P}_{\text {out }}=20 \mathrm{MPa}$, the internal pressure is equal to 0 . The hydrogen concentration on the inner and outer surfaces is kept constant, but with different values. The concentration field of hydrogen is shown in Fig. 9. The intensity of the stresses is shown in Fig. 10. The radial, tangential and axial stresses at time $\mathrm{t}=10$ are shown in Figs 11, 12.13 .

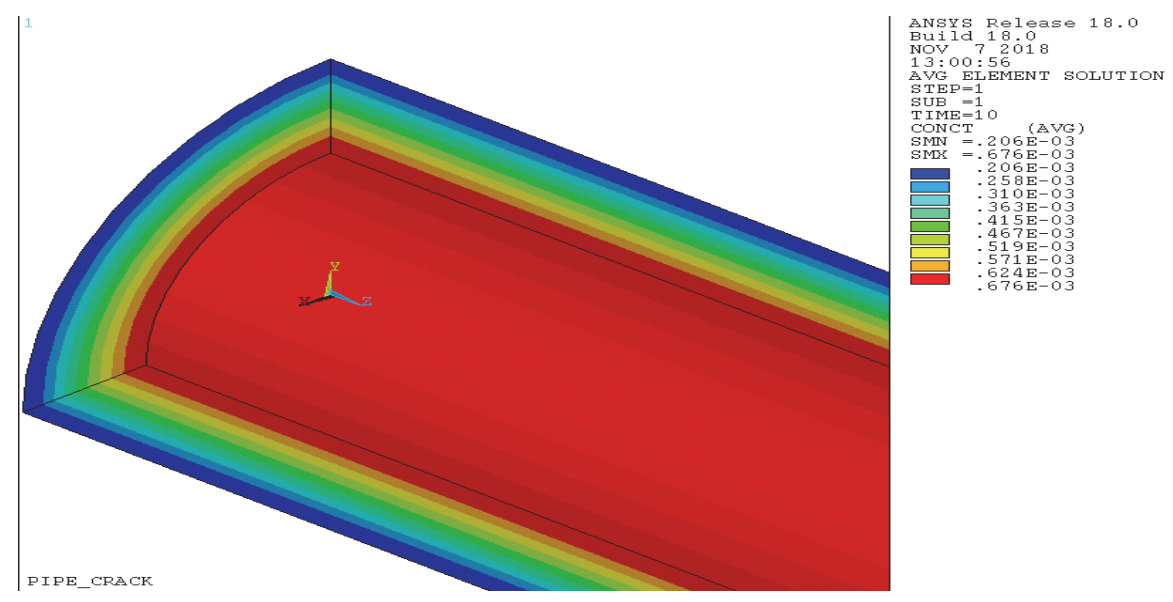

Figure 9: Concentration field of hydrogen.

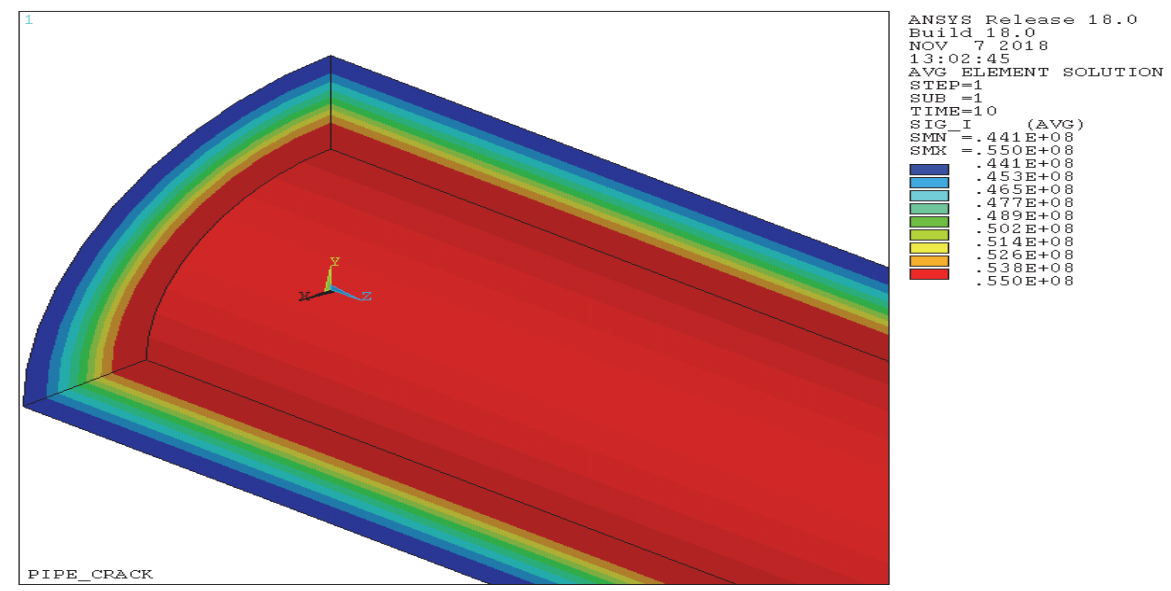

Figure 10: Intensity of constraints at the moment $t=10$.

In Fig. 14, the stress intensity curves $S_{I N T}$, the axial stresses $S_{Z}$, radial and tangential stresses $S_{X}$ and $S_{Y}$ are presented according to the thickness of the wall of the cylinder at time $t=10$.

Note that the deformation pattern does not change over time; however, it is individual for each finite element due to the fact that the concentration field is not uniformly distributed in the volume of the structural element.

The graphs show a change in the character of the stress curves. The redistribution of the tangential stresses and, consequently, of the stress intensities is particularly remarkable.

Loading №3. The loading is similar to the load №2, only the external pressure is $\mathrm{P}_{\text {out }}=30 \mathrm{MPa}$.

The concentration field is stationary and is shown in Fig. 15. The intensity of the stresses is shown in Fig. 16.

The radial, tangential and axial stresses at time $t=18$ are presented in Figs. 17, 18, 19.

In Fig. 20, the stress intensity curves $\mathrm{S}_{\mathrm{INT}}$, the axial stresses $\mathrm{S}_{\mathrm{Z}}$, radial and tangential stresses $\mathrm{S}_{\mathrm{X}}$ and $\mathrm{S}_{\mathrm{Y}}$ are presented according to the thickness of the wall of the cylinder at time $t=18$. 


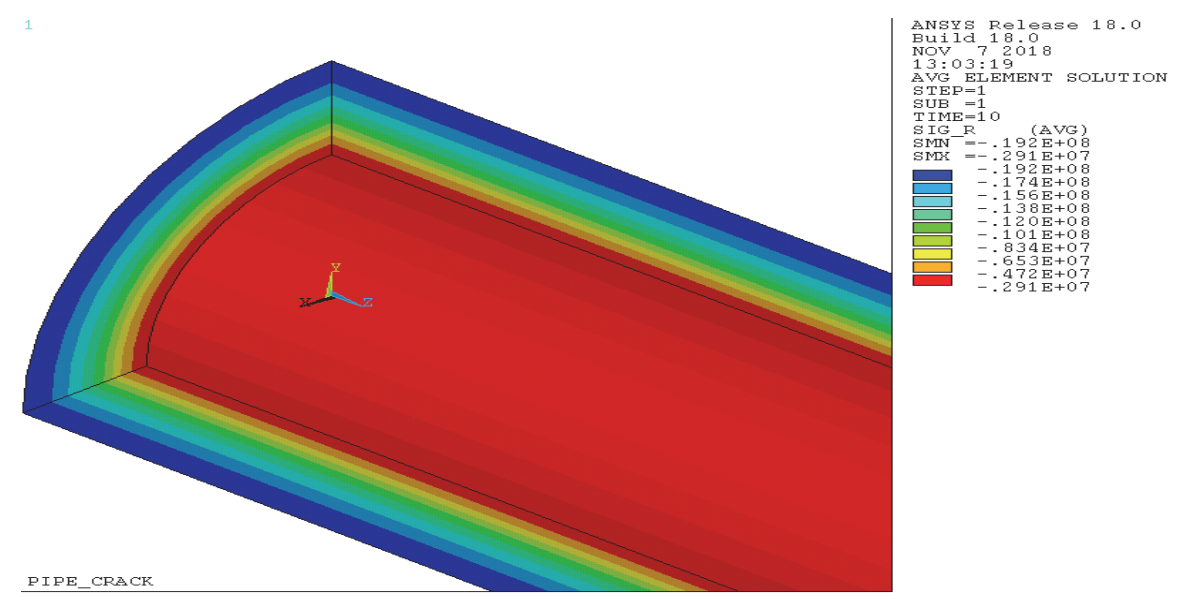

Figure 11: Radial constraints at the moment $t=10$.
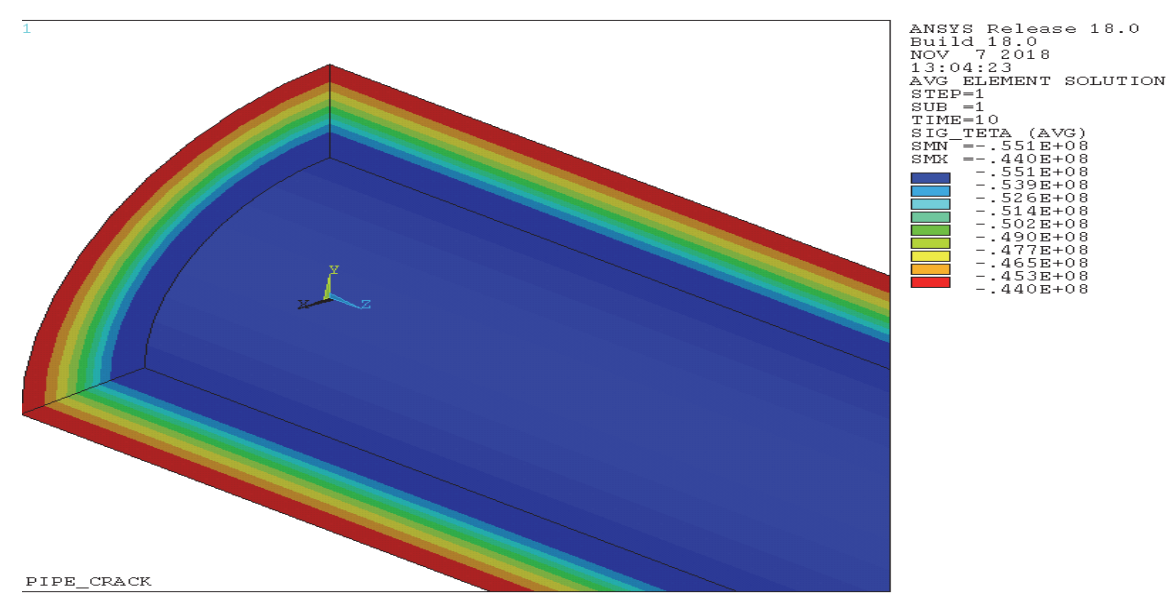

Figure 12: Tangential constraints at the moment $t=10$.

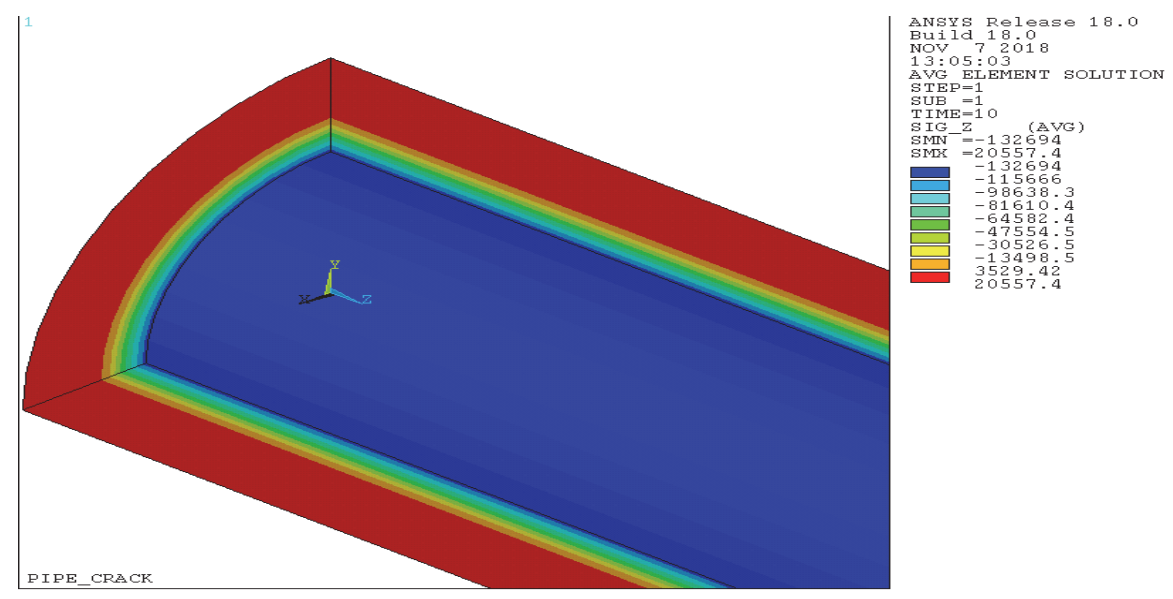

Figure 13: Axial constraints at the moment $t=10$. 


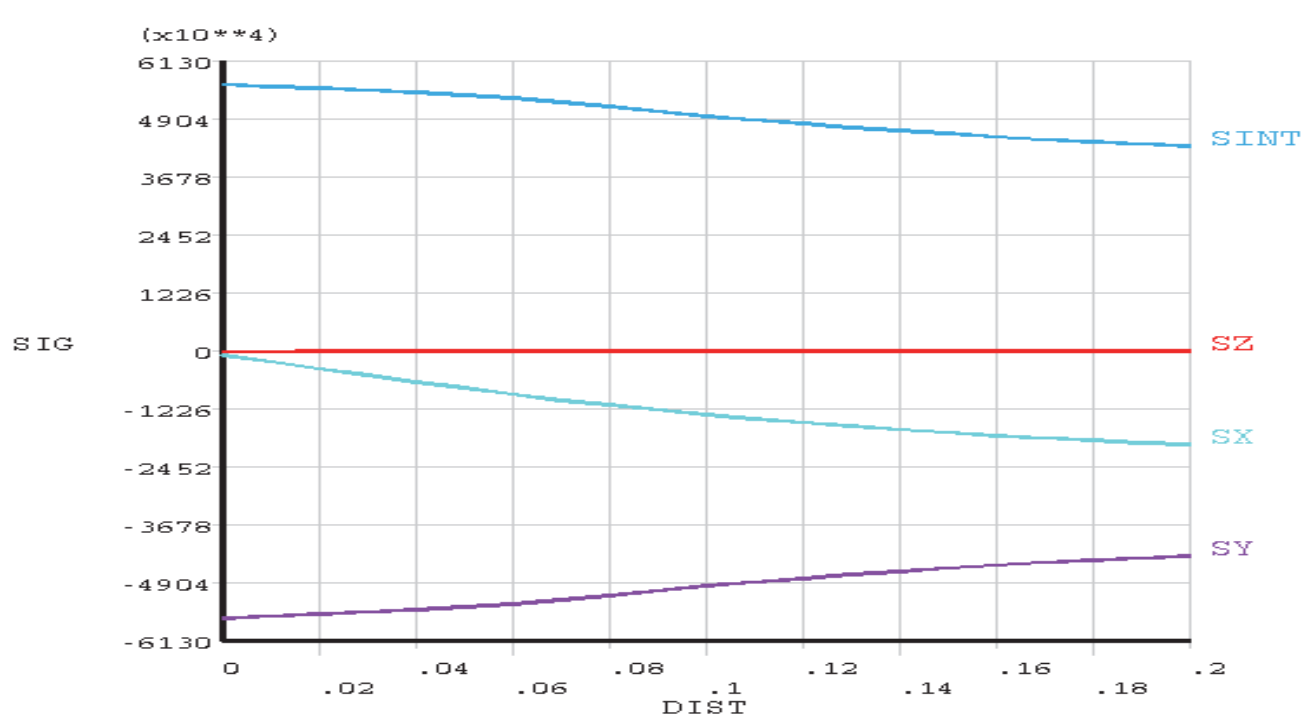

Figure 14: Stress curves according to the thickness at the moment $t=10$.
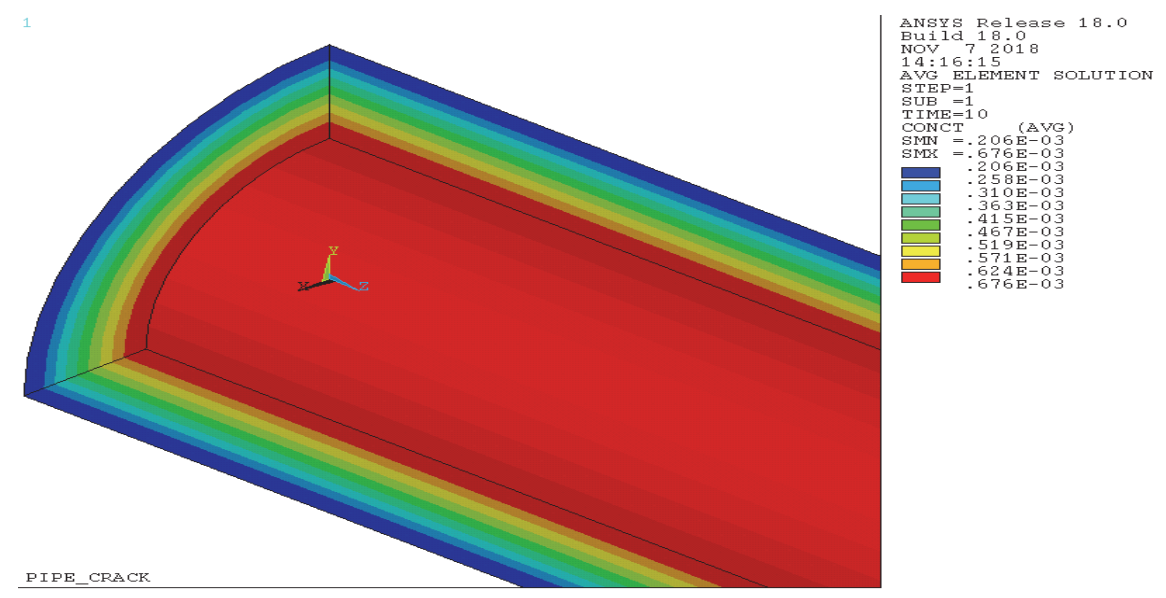

Figure 15: Concentration field of hydrogen.

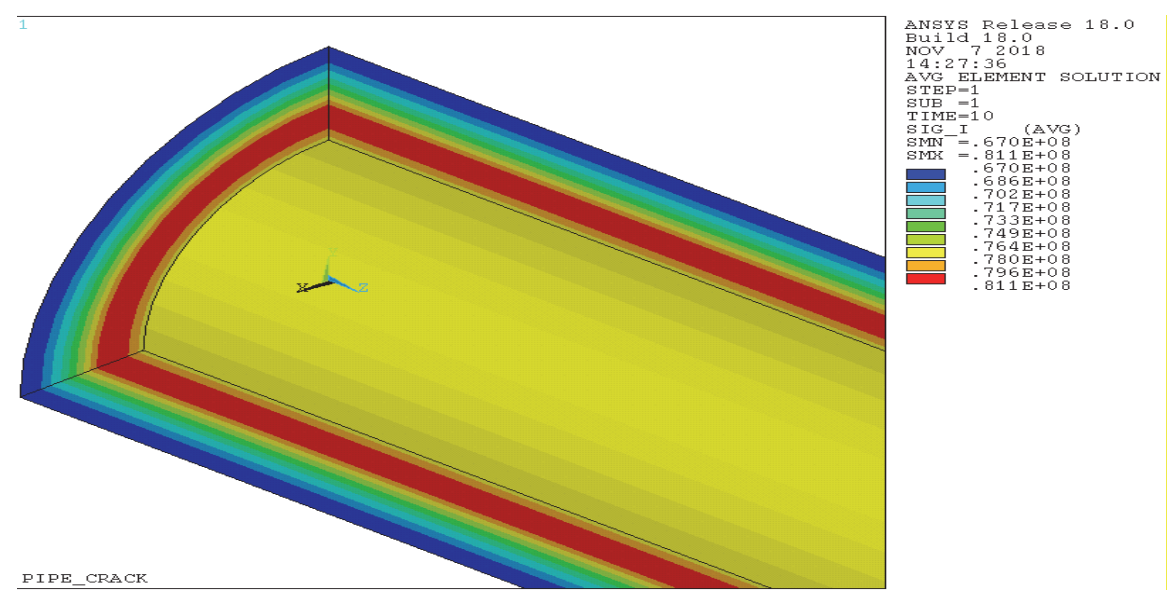

Figure 16: Intensity of constraints at the moment $t=18$. 


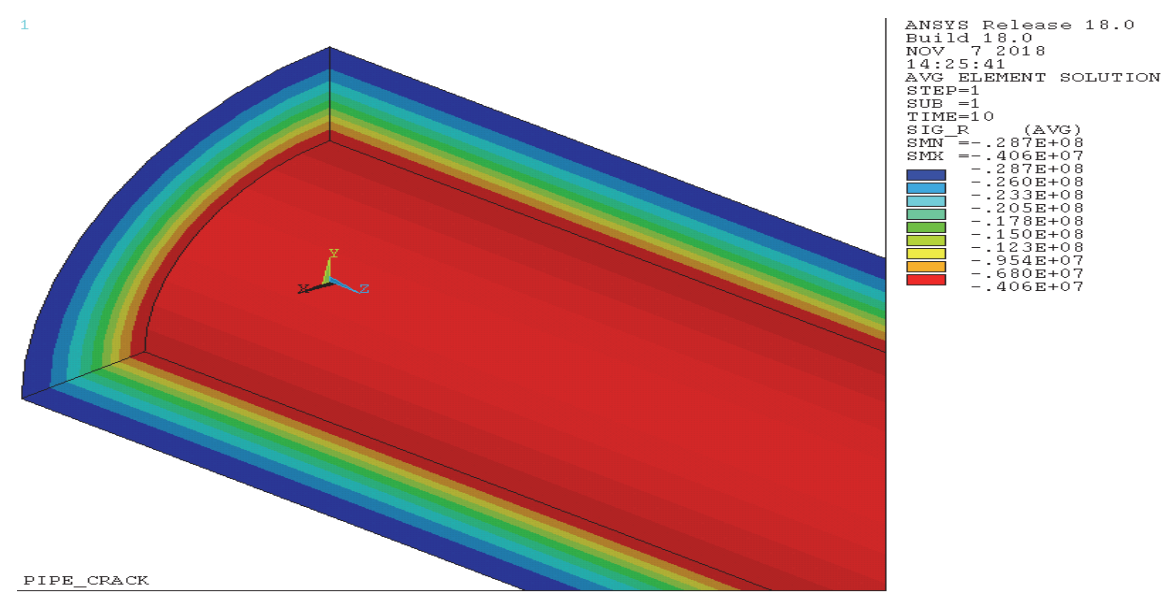

Figure 17: Radial constraints at the moment $t=18$.

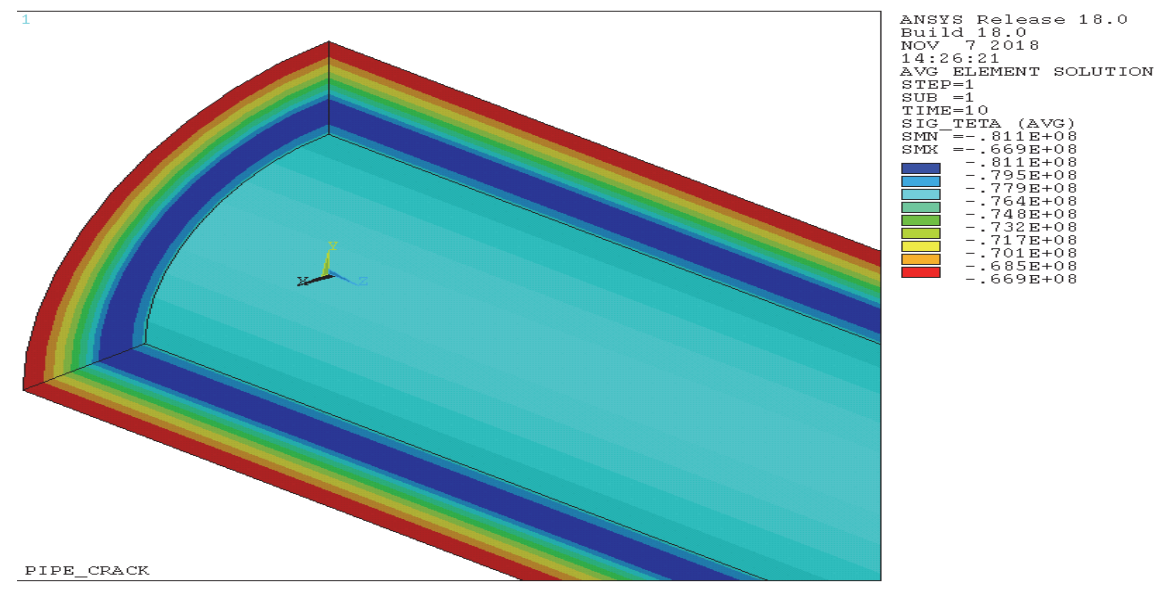

Figure 18: Tangential constraints at the moment $t=18$.
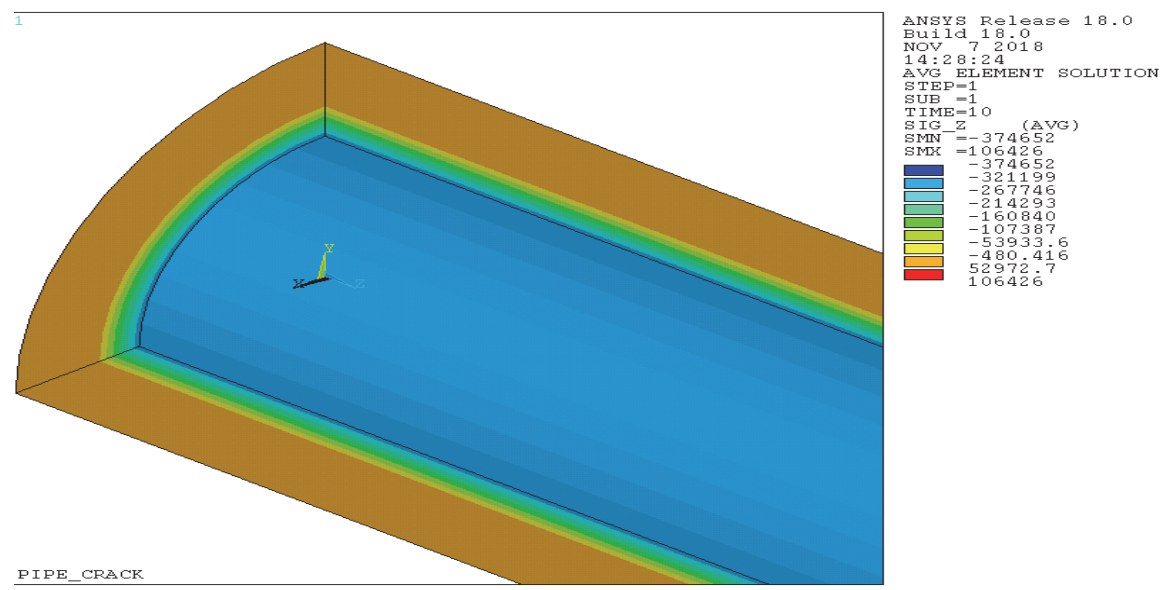

Figure 19: Axial constraints at the moment $t=18$. 
In this case, the redistribution of the constraints is also observed.

Loading №4. The hollow cylinder is subjected to the internal and external pressures $P_{\text {in }}$ and $P_{\text {out }}$ respectively, and $P_{\text {in }}>P_{\text {out }}$, the concentration of hydrogen on the outer surface is constant, and on the inside - it increases linearly with each time step from a certain initial value.

As a result, we obtain a time-varying concentration field (Figs. 21 and 22).

The intensity of the stresses is shown in Fig. 23. The radial, tangential and axial stresses at time $t=11$ are presented in Figs. 24, 25, and 26.

There is also a redistribution of the constraints, and more intense than in the cases of loading previously envisaged. In Fig. 27, the stress intensity curves $S_{\text {INT, }}$, the axial stresses $S_{Z}$, radial and tangential stresses $S_{X}$ and $S_{Y}$ are presented according to the thickness of the wall of the cylinder at time $t=11$.

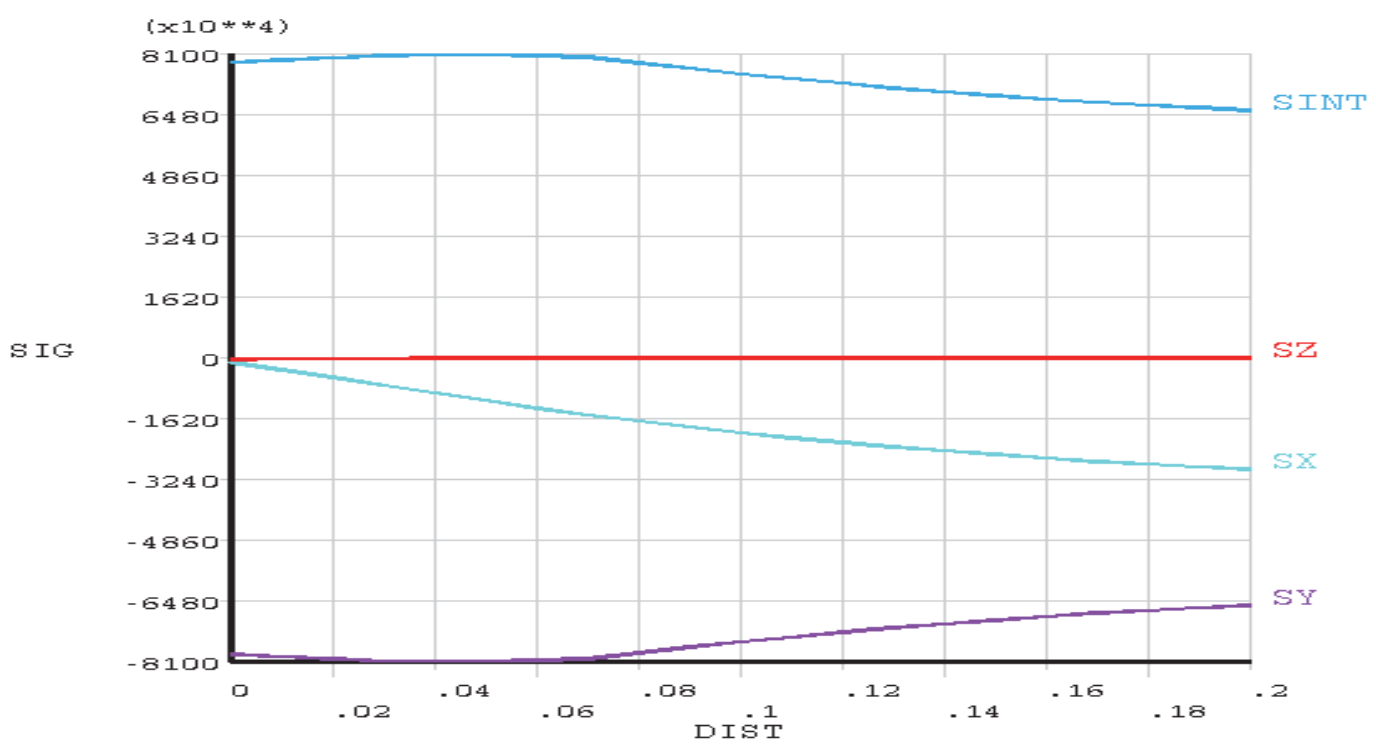

Figure 20: Stress curves according to the thickness at the moment $t=18$.

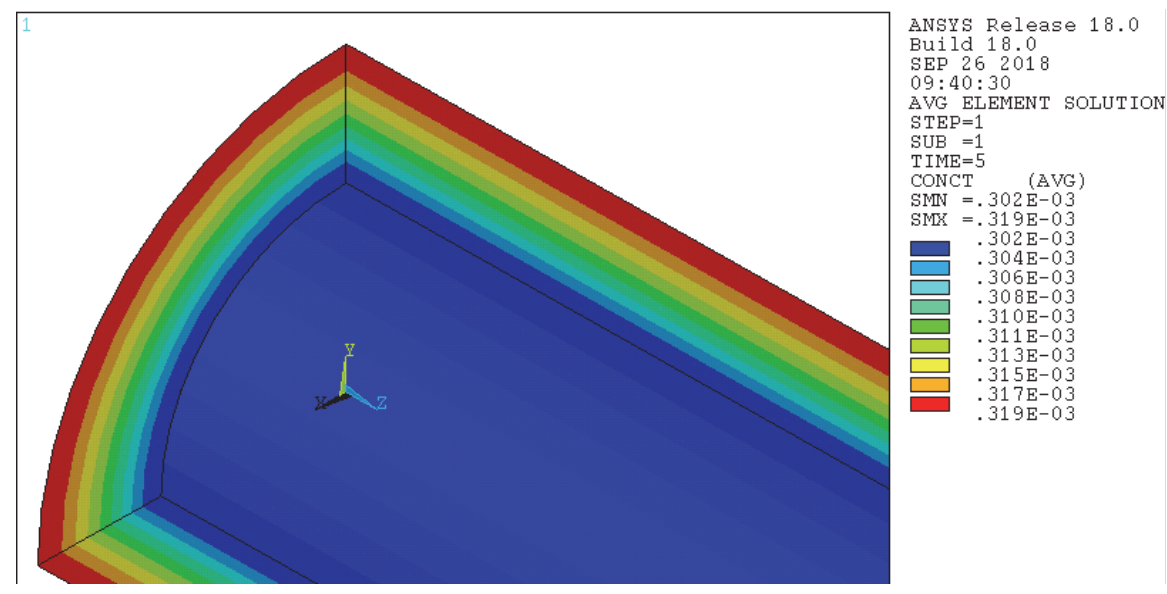

Figure 21: Concentration field of hydrogen at the moment $t=5$. 


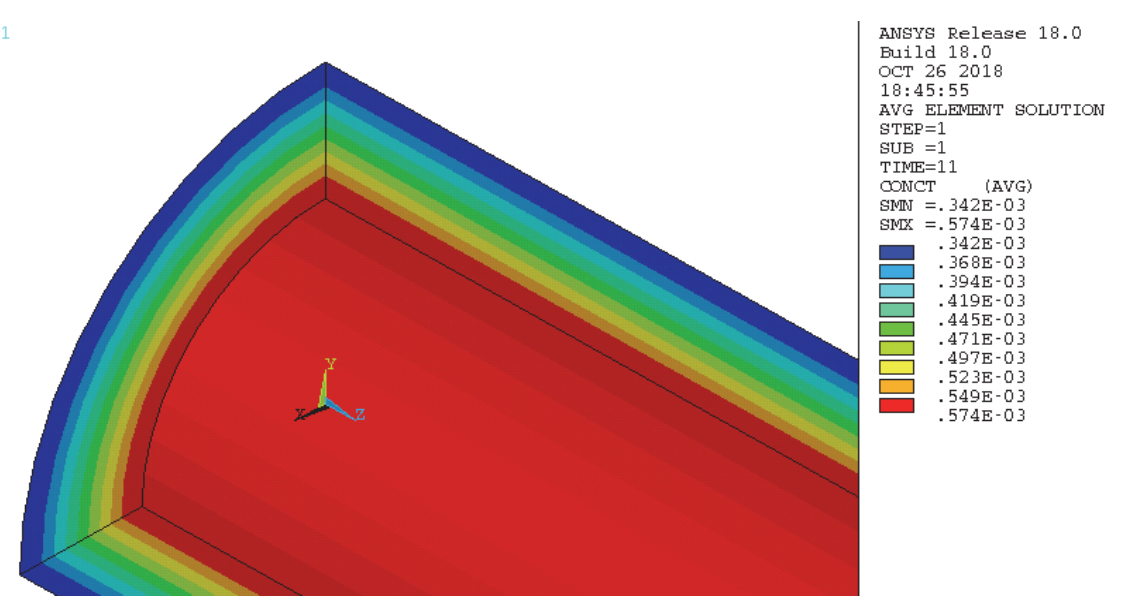

Figure 22: Concentration field of hydrogen at the moment $t=11$.

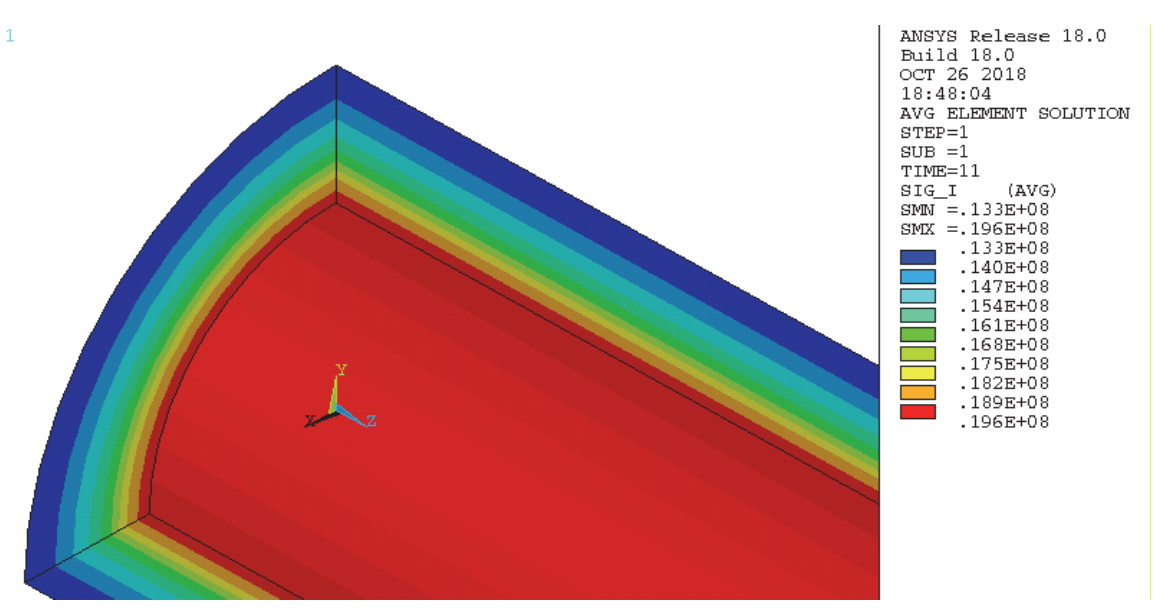

Figure 23: Intensity of constraints at the moment $t=11$.

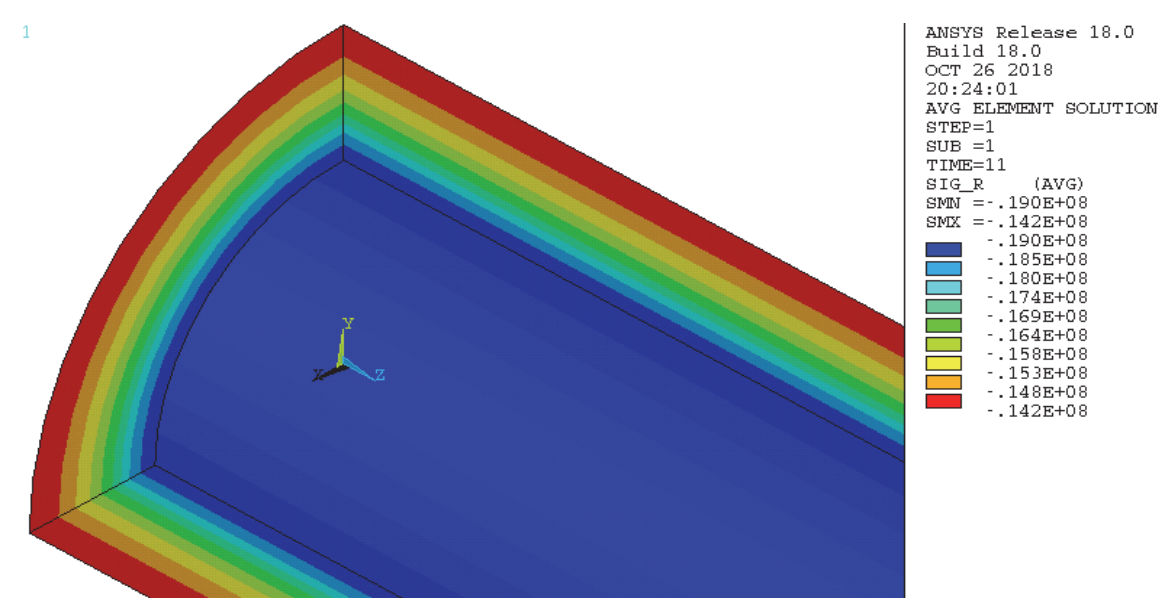

Figure 24: Radial constraints at the moment $t=11$. 


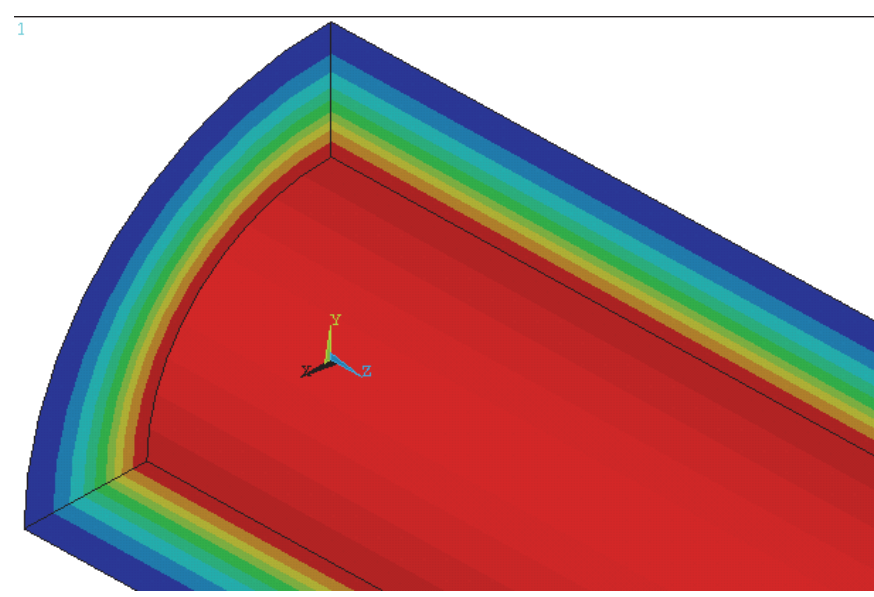

ANSYS Release 18.0

Build 18.0
OCT $26 \quad 2018$

$20: 25: 50$

AVG ELEMENT SOLUTION

STEP $=1$
SUB $=1$

TIME $=11$

SIG_TETA (AVG)

SMIN $=-.701 \mathrm{E}+07$

$-.701 \mathrm{E}+07$

$-.647 \mathrm{E}+07$
$-.593 \mathrm{E}+07$
$-.539 \mathrm{E}+07$

$-.539 \mathrm{E}+07$
$-.486 \mathrm{E}+07$
$-.432 \mathrm{E}+07$

$.432 \mathrm{E}+07$

$324 \mathrm{E}+07$

$.217 \mathrm{E}+07$

Figure 25: Tangential constraints at the moment $t=11$.

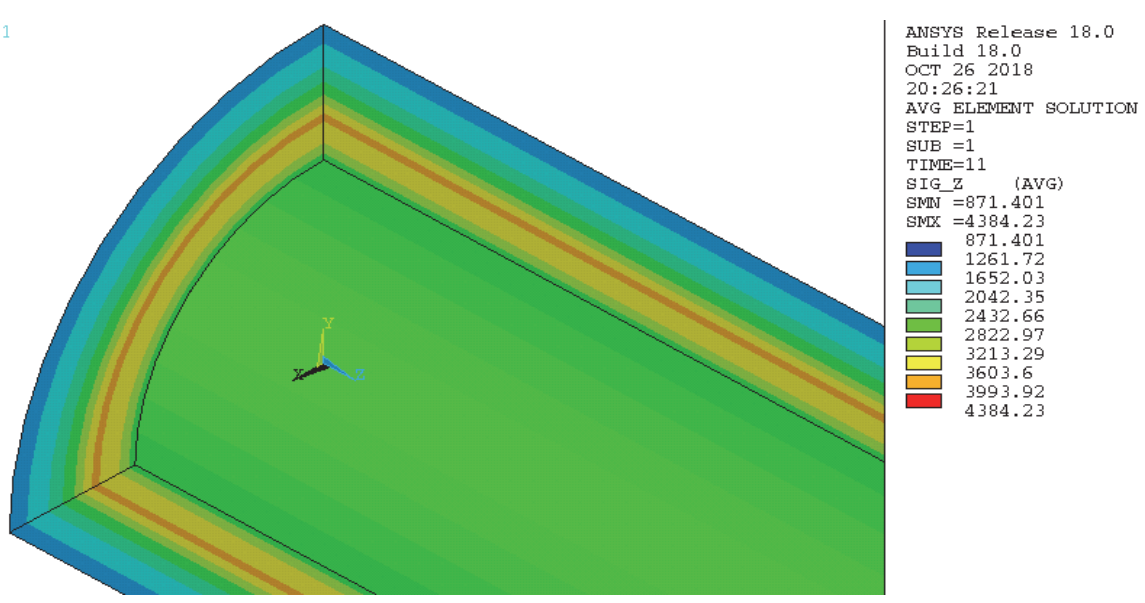

Figure 26: Axial constraints at the moment $t=11$.

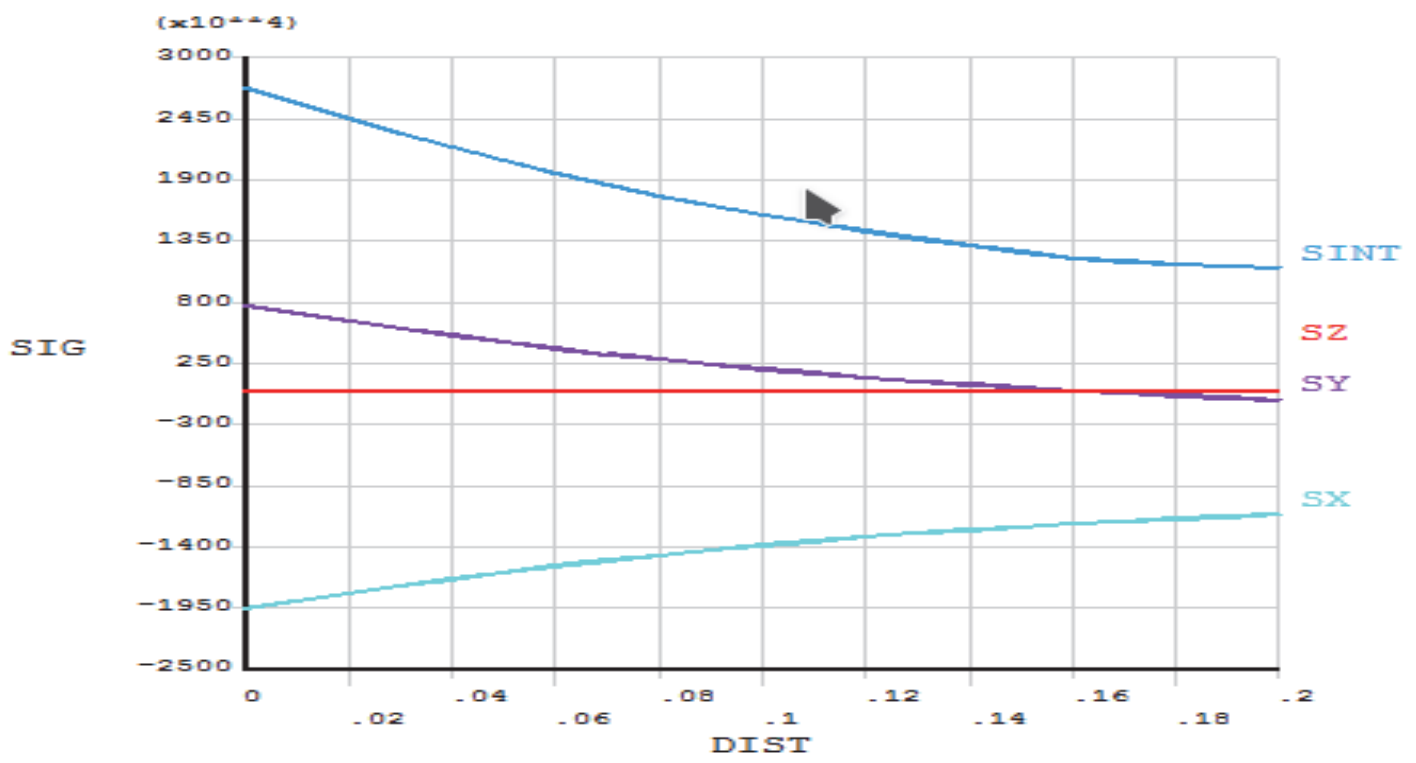

Figure 27: Stress curves according to the thickness at the moment $t=11$. 


\section{CONCLUSIONS}

1. The study showed that the use of the finite element software ANSYS allows numerical simulations of the changes in the hydrogen concentration field and the stress-strain state of a hollow cylinder, not only the action of hydrogen at high pressure and temperature, as has been as done in the work [16], but also when hollow cylinder interacts with hydrogen at low temperature.

2. Adapted to the resolution of such problems, the ANSYS software package is used to model the behavior of different structures in a hydrogen-containing environment, taking into account the effects caused by both the influence of hydrogen on the mechanical properties of hydrogen material and stress state of structures, and considering the influence of state of stress on the kinetics of the interaction of hydrogen with structures.

3. The elaborate model of material behavior of structural elements in a hydrogenated medium takes into account the selective effect of low temperature hydrogen on the mechanical properties of materials. The selectivity is expressed as a function of the anisotropy induced by the hydrogen concentration and the rigidity of the stress state scheme.

4. The proposed model takes into account the influence of the type and the level of the stress state of the material on the kinetics of hydrogen penetration into the material by the dependence of the diffusion coefficient and the absorption limit of the material. hydrogen.

5.It should be noted that the established relationships fairly adequately describe the behavior of the tube under the combined action of charge and hydrogenation, while taking into account the destructive action of hydrogen.

6. The numerical simulation shows that the most dangerous case is the case of the simultaneous action of the charge and the hydrogen on the inner surface of the wall of the tube, because in this case the combination of the action of the stresses of traction and hydrogen leads to an intensive degradation of the tube material.

7.It should be noted that, contrary to the case of hydrogen at high pressure and temperature, the penetration of hydrogen occurs through the mechanism of activated diffusion and that the kinetics of hydrogen penetration depends on the rigidity of the scheme of the state of constraint. Since the state of stress changes with time under the influence of hydrogen entering the structure, the kinetics of hydrogen penetration into the structural element also changes over time.

8. Hydrogen embrittlement of metal structures is closely related to their microstructure, and in particular to the processes of segregation and diffusion occurring at interfaces and defects.

\section{REFERENCES}

[1] Lynch, S. (2019). Discussion of some recent literature on hydrogen-embrittlement mechanisms: addressing common misunderstandings, Corrosion Reviews; 37(5), pp. 377-395. DOI: 10.1515/corrrev-2019-0017.

[2] Djukic, M.B., Bakic, G.M., Sijacki Zeravcic, V., Sedmak, A., Rajicic, B. (2016). Hydrogen Embrittlement of Industrial Components: Prediction, Prevention, and Models, Corrosion; 72 (7), pp. 943-961. DOI: 10.5006/1958.

[3] Bueno, A., Moreira, E., Gomes, J. (2014). Evaluation of stress corrosion cracking and hydrogen embrittlement in an API grade steel, Engineering Failure Analysis; 36, pp. 423-431. DOI: 10.1016/j.engfailanal.2013.11.012.

[4] Serebrinsky, S., Carter, E.A., Ortiza, M. (2004). A quantum mechanically informed continuum model of hydrogen embrittlement, J. of the Mechanics and Physics of Solids; 52 (10), pp. $2403-2430$.

DOI: $10.1016 /$ j.jmps.2004.02.010.

[5] Kolachev, B.A. (1999). Hydrogen in metals and alloys, Metal Science and Heat Treatment; 41(3), pp. 93-100. DOI: $10.1007 / \mathrm{BF} 02467692$.

[6] Woodtli, J., Kieselbach, R. (2000). Damage due to hydrogen embrittlement and stress corrosion cracking, Eng. Failure Analysis; 7, pp. 427- 450. DOI: 10.1016/S1350-6307(99)00033-3.

[7] Lynch, S. (2012). Hydrogen embrittlement phenomena and mechanisms, Corrosion Reviews; 30(3-4), pp. 105-123. DOI: $10.1515 /$ corrrev-2012-0502.

[8] May, L.M., Dadfarnia, M., Nagao, A., Wang, S., Sofronis, P. (2019). Enumeration of the hydrogen-enhanced localized plasticity mechanism for hydrogen embrittlement in structural materials, Acta Materialia; 165, pp. 734-750.

DOI: doi.org/10.1016/j.actamat.2018.12.014.

[9] Djukic, M.B., Bakic, G.M., Zeravcic, V. S., Sedmak, A., Rajicic, B. (2019). The synergistic action and interplay of hydrogen embrittlement mechanisms in steels and iron: Localized plasticity and decohesion, Eng. Fracture Mechanics; 216, pp. 106-528. DOI: 10.1016/j.engfracmech.2019.106528. 
[10] Djukic, M.B., Sijacki Zeravcic, V., Bakic, G.M., Sedmak, A., Rajicic, B. (2015). Hydrogen damage of steels: A case study and hydrogen embrittlement model, Eng. Failure Analysis; 58 (2), pp. 485-498.

DOI: $10.1016 /$ j.engfailanal.2015.05.017.

[11] Ovchinnikov, I.G. and Khvalko, T.A. (2003). Serviceability of structures under high-temperature hydrogen corrosion, ed. Sarat. Gos. Tekhn. Un-t. Saratov, Russia.

[12] Balueva, A. (2014). Modeling of Hydrogen Embrittlement Cracking in Pipe-lines under High Pressures, Procedia Materials Science.; 3, pp. 1310-1315. DOI: doi.org/10.1016/j.mspro.2014.06.212.

[13] Hardie, D., Charles, E.A., Lopez, A.H. (2006). Hydrogen embrittlement of high strength pipeline steels; Corrosion Science. ; 48 (12), pp. 4378-4385. DOI: 10.1016/j.corsci.2006.02.011.

[14] Novak, P., Yuan, R., Somerday, B.P., Sofronis, P., Ritchie, R.O. (2010). A statistical, physical-based, micro-mechanical model of hydrogen-induced intergranular fracture in steel, J. of Mechanics and Physics of Solids; 58 (2), pp. 206226. DOI: 10.1016/j.jmps.2009.10.005.

[15] Kim, N.H., Oh, C.S., Kim, Y.J., Yoon, Ke, B., Ma, Y.W. (2012). Hydrogen-assisted stress corrosion cracking simulation using the stress-modified fracture strain model, J. of Mechanical Science and Technology; 26 (8), pp. 2631-2638. DOI 10.1007/s12206-012-0642-x.

[16] Bubnov, A.A., Bubnov, S.A. and Ovchinnikov, I. I. (2011). Modelling of stress state and fracture of thick-walled piping in hydrogen corrosion and heterogeneous thermal field, ed. Goryachaya Liniya -Telekom. Moscow, Russia.

[17] Ovchinnikov, I.I. (2013). Models of deformation and delayed destruction of materials in a hydrogen-containing environment, Vestnik SSTU; 2 (70), pp. 178-183. (Russian)

[18] Ovchinnikov, I.I. (2013). Modeling the behavior of a long hollow cylinder in a hydrogen-containing environment, the diffusion characteristics of which depend on the stressed state, Vestnik SSTU; 2 (70), pp. 183-191. (Russian)

[19] ANSYS Element Reference. ANSYS Release 18.0. Documentation Inc., 2017.

[20] ANSYS Theory Reference. ANSYS Release 18.0. Documentation Inc., 2017.

[21] ANSYS Parametric Design Language Guide. ANSYS Release 18.0. Documentation Inc., 2017.

[22] ANSYS Modeling and Meshing Guide. ANSYS Release 18.0. Documentation Inc., 2017. 